\title{
Delayed versus Delayed-Immediate Autologous Breast Reconstruction: A Blinded Evaluation of Aesthetic Outcomes
}

\author{
Frank P Albino, Ketan M Patel, Jesse R Smith, Maurice Y Nahabedian \\ Department of Plastic Surgery, Georgetown University Hospital, Washington, DC, USA
}

Background The technique of delayed-immediate breast reconstruction includes immediate insertion of a tissue expander, post-mastectomy radiation, followed by reconstruction. The aesthetic benefits of delayed-immediate reconstruction compared to delayed reconstruction are postulated but remain unproven. The purpose of this study was to compare aesthetic outcomes in patients following delayed and delayed-immediate autologous breast reconstruction.

Methods A retrospective analysis was performed of all patients who underwent delayed or delayed-immediate autologous breast reconstruction by the senior author from 2005 to 2011. Postoperative photographs were used to evaluate aesthetic outcomes: skin quality, scar formation, superior pole contour, inferior pole contour, and overall aesthetic outcome. Ten non-biased reviewers assessed outcomes using a 5-point Likert scale. Fisher's Exact and Wilcoxon-Mann-Whitney tests were used for comparative analysis.

Results Patient age and body mass index were similar between delayed $(n=20)$ and delayedimmediate $(n=20)$ cohorts $(P>0.05)$. Skin and scar quality was rated significantly higher in the delayed-immediate cohort ( 3.74 vs. $3.05, \mathrm{P}<0.001$ and 3.41 vs. $2.79, \mathrm{P}<0.001$; respectively). Assessment of contour-related parameters, superior pole and inferior pole, found significantly improved outcomes in the delayed-immediate cohort (3.67 vs. $2.96, \mathrm{P}<0.001$ and 3.84 vs. 3.06, $\mathrm{P}<0.001$; respectively). Delayed-immediate breast reconstruction had a significantly higher overall score compared to delayed breast reconstructions ( 3.84 vs. $2.94, \mathrm{P}<0.001$ ). Smoking and the time interval from radiation to reconstruction were found to affect aesthetic outcomes $(\mathrm{P}<0.05)$.

Conclusions Preservation of native mastectomy skin may allow for improved skin/scar quality, breast contour, and overall aesthetic outcomes following a delayed-immediate reconstructive algorithm as compared to delayed breast reconstruction.

Keywords Mammaplasty / Breast implantation / Cosmetic techniques / Mastectomy

\author{
Correspondence: Frank P Albino \\ Department of Plastic Surgery, \\ Georgetown University Hospital, 1st \\ Floor PHC Building, 3800 Reservoir \\ Road, N.W., Washington, DC 20007, \\ USA \\ Tel: +1-973-727-9787 \\ Fax: +1-202-444-1233 \\ E-mail: Frankalbino@gmail.com
}

This article was presented at the Northeastern Society of Plastic Surgeons on September 20-22, 2013 in Washington, DC, USA.

No funding was utilized for the preparation of this manuscript. Dr. Nahabedian is a member of the Speakers Bureau for LifeCell Corporation (Branchburg, New Jersey). No other authors have relevant conflicts of interest or financial disclosures.

Received: 27 Jan 2014 • Revised: 25 Feb 2014 • Accepted: 6 Mar 2014

pISSN: 2234-6163 • elSSN: 2234-6171 • http://dx.doi.org/10.5999/aps.2014.41.3.264 • Arch Plast Surg 2014;41:264-270

\section{INTRODUCTION}

Despite a proven oncologic benefit in select patients, post-mas- tectomy radiation therapy (PMRT) will often have a negative impact on aesthetic outcomes following breast reconstruction [1-3]. Reported adverse effects include but are not limited to

Copyright $(\odot 2014$ The Korean Society of Plastic and Reconstructive Surgeons

This is an Open Access article distributed under the terms of the Creative Commons Attribution Non-Commercial License (http://creativecommons.org/

licenses/by-nc/3.0/) which permits unrestricted non-commercial use, distribution, and reproduction in any medium, provided the original work is properly cited. 
capsular contracture, breast distortion, infection, parenchymal atrophy, and fat necrosis [4]. Efforts to better understand the relationship between these radiation related morbidities and various patient/treatment factors have been investigated in an effort to improve surgical and aesthetic outcomes. The nature of the reconstruction has also been postulated to influence outcomes following radiation therapy. Adverse events may be more pronounced following device-based reconstruction when compared to autologous-based reconstruction in the setting of radiation therapy $[5,6]$. Although, autologous reconstruction appears to tolerate radiation better than prosthetic reconstructions with a lower incidence of major corrective surgery, the late adverse events associated with autologous reconstruction are difficult to correct $[6,7]$. In addition, autologous reconstructions have a decreased rate of reconstructive failure when compared to devicebased reconstructions alone [8].

The timing of breast reconstruction relative to PMRT is another important factor impacting outcomes. With the anticipation of radiotherapy, immediate (pre-radiation) breast reconstructions using autologous tissues or prosthetic devices may be associated with higher rates of complications [9]. Despite some reported success [5], immediate autologous reconstruction has been criticized for compromising delivery of PMRT and facilitating flap related adverse events such as skin contracture, parenchymal induration, fat necrosis, and hyperpigmentation. These outcomes may compromise breast symmetry and often lead to aesthetically inconsistent results $[10,11]$. Post-radiation changes are often difficult to correct, may require multiple revision procedures, and can adversely affect aesthetic outcomes.

There are many women that desire autologous reconstruction but may require post mastectomy radiation therapy. The question is whether to perform the flap immediately following the mastectomy or in a delayed basis following the radiation. The advantage of immediate reconstruction is that the skin envelope is preserved and aesthetic outcomes are usually enhanced. The advantage of delayed reconstruction is that there is no impedance to radiation delivery and it does not impede recurrence detection nor does it affect overall survival in the event of local regional recurrence [3].

In an effort to preserve native breast skin, improve aesthetic outcomes, and minimize challenges associated with radiation delivery, the concept of delayed-immediate reconstruction (DIR) was proposed by Fine and reported by Kronowitz et al. [12] in 2004. When radiation therapy is anticipated, a tissue expander is inserted to preserve the mastectomy skin envelope and to maintain the breast pocket. The volume of the tissue expander can be adjusted as needed during the radiation delivery. Following radiotherapy, the tissue expander is removed and replaced with autologous tissue. The advantage of this approach is that the flap will remain soft and supple without the associated changes typically seen with autologous tissue that has been directly radiated; while at the same time, radiation delivery is not impeded.

With established safety and efficacy of the DIR algorithm [13], it is important to consider whether aesthetic outcomes are actually improved using this algorithm. To date, no study has directly compared aesthetic outcomes utilizing objective measures and multiple, blinded raters. The purpose of this study was to investigate the aesthetic outcomes of both delayed and DIR in patients receiving postmastectomy radiation therapy.

\section{METHODS}

An Institutional Review Board-approved, retrospective analysis was performed of patients who underwent autologous breast reconstruction by the senior author (M.Y.N.) from 2005 to 2011. Medical charts were reviewed to identify all patients who underwent autologous reconstruction in a delayed or delayed immediate fashion following mastectomy. Evaluating only those patients that had radiation therapy, the total number of delayed reconstructions was 99 and the total number of delayed immediate reconstructions was 53 .

Medical records were reviewed for each of these 106 patients to collect demographic, operative, and postoperative data. Photographs routinely taken at each office visit prior to and after all surgical procedures were obtained; however, only 42 patients had documented 3-view photographs taken at least 6-months following surgery. Of these 42 reconstructions, 20 patients underwent a unilateral, delayed autologous breast reconstruction and 20 patients underwent a unilateral, DIR. Frontal photographs for each of these 40 patients were arranged in an arbitrary order for a slideshow consisting of both the delayed and delayed immediate cohorts. Fig. 1 demonstrates representative, singleview photographs taken 6 months following DIR while Fig. 2 depicts the same following delayed reconstructions. Images were displayed, one at a time, by an individual who had no knowledge of the patients and presented to 10 non-biased reviewers who also had no knowledge of the patients. These reviewers consisted of pediatric nurses who were asked to evaluate the selected outcome parameters using a 5-point Likert scale. The outcome parameters included skin quality, scar formation, superior pole contour, inferior pole contour, and overall aesthetic outcomes (Table 1). Following the administration of the survey, scores were linked to each corresponding patient. Data were pooled for each cohort to compare outcomes between treatment arms. Statistical Analysis Software was used to conduct two-tailed Student's T, Fisher's Exact, and Wilcoxon-Mann-Whitney tests as a 


\section{Fig. 1. Delayed-immediate reconstruction}

Frontal photographs taken six-months following delayed-immediate reconstructions prior to nipple reconstruction for patients who received the highest (A), average (B), and lowest (C) scores within this cohort.
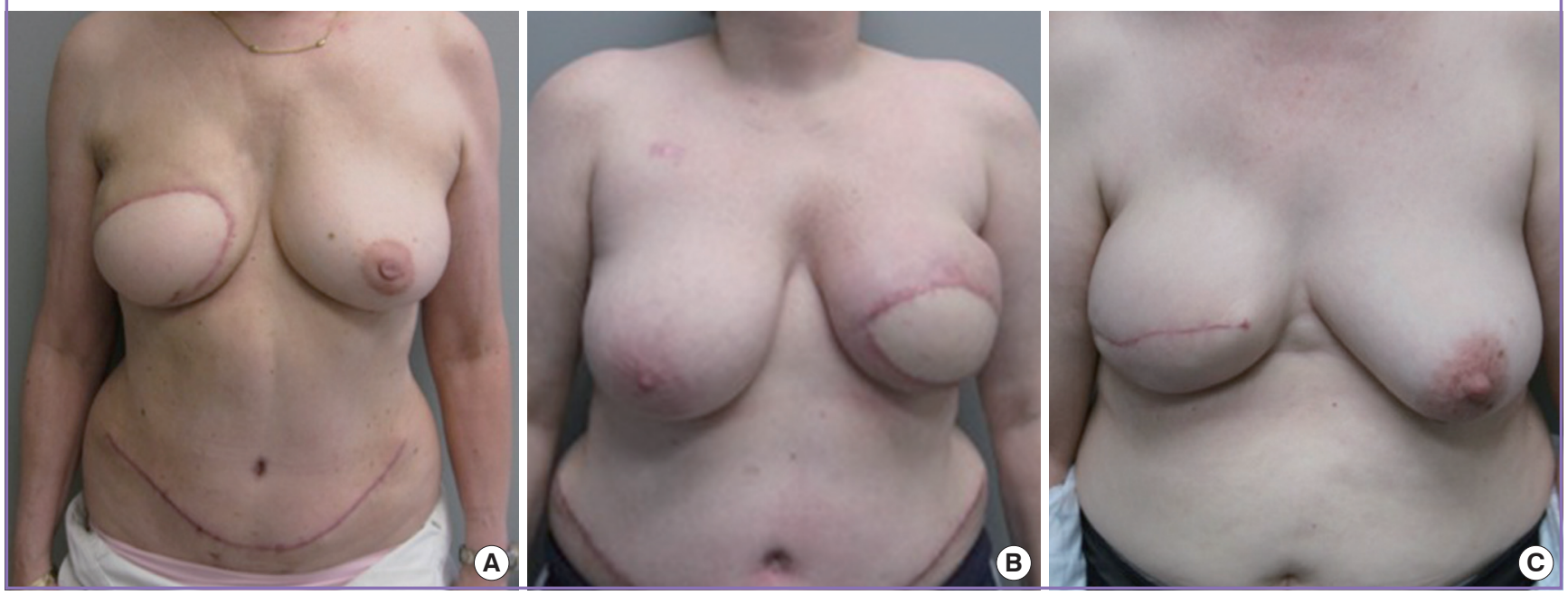

Fig. 2. Delayed reconstruction

Frontal photographs taken six-months following delayed reconstructions prior to nipple reconstruction for patients who received the highest (A), average (B), and lowest (C) scores within this cohort.

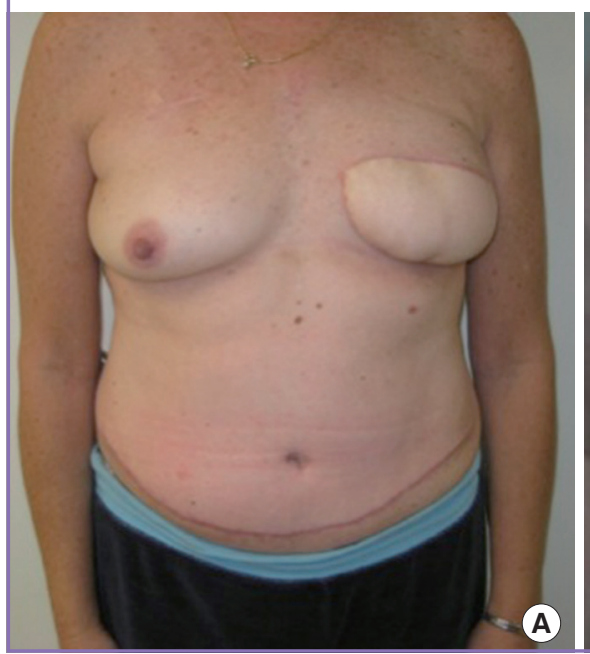

comparative analysis between outcomes and treatment factors.

\section{RESULTS}

Patient demographics were not significantly different between the delayed $(n=20)$ and delayed immediate $(n=20)$ patient cohorts (Table 2 ). The mean patient age was 51 years with a mean body mass index (BMI) of $28 \mathrm{~kg} / \mathrm{m}^{2}$. Medical co-morbidities including diabetes mellitus and coronary artery disease were similar between treatment groups (1.4 in the delayed reconstruction [DR] vs. 1.5 in the DIR). Hypertension was the most common secondary diagnosis with an overall prevalence of $60 \%(12 / 20$ patients). Autologous reconstructions consisted of 24 deep in-
Table 1. Administered survey

\begin{tabular}{lccccc|}
\hline Variable & Very poor & Poor & Average & Good & Very good \\
\hline Skin quality & 1 & 2 & 3 & 4 & 5 \\
Scar quality & 1 & 2 & 3 & 4 & 5 \\
Superior pole contour & 1 & 2 & 3 & 4 & 5 \\
Inferior pole contour & 1 & 2 & 3 & 4 & 5 \\
Overall outcome & 1 & 2 & 3 & 4 & 5 \\
\hline
\end{tabular}

Breast Reconstruction Aesthetic Survey. Each of the following patients underwent an autologous breast reconstruction following mastectomy. Please evaluate only the reconstructed breast for each patient. Do not consider nipple placement or abdominal scar.

ferior epigastric perforator flap (DIEPs) and 16 muscle-sparing free transverse rectus abdominis myocutaneous (TRAM) flaps. 
Table 2. Patient demographics

\begin{tabular}{|llcc|}
\hline Variable & $\begin{array}{c}\text { Delayed } \\
\text { cohort (20) }\end{array}$ & $\begin{array}{c}\text { Delayed-immediate } \\
\text { cohort (20) }\end{array}$ & P-value \\
\hline Age (yr, SD) & $49.9(9.1)$ & $52.5(10.2)$ & 0.62 \\
Body mass index (kg/m², SD) & $32.2(6.2)$ & $27.1(6.6)$ & 0.08 \\
No. of comorbidities (SD) & $1.44(0.73)$ & $1.55(1.2)$ & 0.97 \\
Diabetes mellitus (\%) & $10(2 / 20)$ & $20(4 / 20)$ & 0.99 \\
CAD (\%) & $10(2 / 10)$ & $0(0 / 20)$ & 0.99 \\
Time from mastectomy to & $21(9-60)$ & $10(6-18)$ & 0.04 \\
reconstruction (mo) & & \\
\hline SD, standard deviation; CAD, coronary artery disease. & \\
\hline
\end{tabular}

Table 3. Aesthetic outcomes as related to timing of reconstruction

\begin{tabular}{|lccc|}
\hline Variable & Delayed cohort & Delayed immediate cohort & P-value \\
\hline Skin flap quality & $3.05(0.80)$ & $3.74(0.86)$ & $<0.001$ \\
Scar quality & $2.79(0.77)$ & $3.41(1.04)$ & $<0.001$ \\
$\begin{array}{l}\text { Superior breast } \\
\text { contour }\end{array}$ & $2.96(0.81)$ & $3.67(0.94)$ & $<0.001$ \\
$\begin{array}{l}\text { Inferior breast } \\
\text { contour }\end{array}$ & $3.06(0.86)$ & $3.84(0.90)$ & $<0.001$ \\
Overall & $2.94(0.83)$ & $3.84(0.89)$ & $<0.001$ \\
\hline
\end{tabular}

The mean time interval from mastectomy to final reconstruction was 21 months (range, 9-60 months) in the delayed cohort and 10 months (range, 6-18 months) in the delayed-immediate cohort. Complications following the first-stage of reconstruction were $8.5 \%$ and $10.8 \%$ in the delayed and delayed-immediate cohorts, respectively. In the delayed immediate treatment group there was a $50 \%$ infection rate, $25 \%$ skin necrosis rate, and $25 \%$ seroma rate. Following a mastectomy without tissue expander placement in the delayed cohort, complications included $10 \%$ infection, $20 \%$ skin necrosis, and $40 \%$ seroma.

Based on overall aesthetic outcomes, the delayed-immediate cohort scored statistically superior to the delayed cohort in all 5 items measured by the Likert-scale survey (Table 3). Specific differences included skin and scar quality that was rated significantly higher in the delayed-immediate cohort (3.74 vs. 3.05 , $\mathrm{P}<0.001$ and 3.41 vs. $2.79, \mathrm{P}<0.001$; respectively). Contourrelated parameters related to superior and inferior poles were significantly improved in the delayed-immediate cohort (3.67 vs. $2.96, \mathrm{P}<0.001$ and 3.84 vs. $3.06, \mathrm{P}<0.001$; respectively). When graded purely on overall aesthetic score, the delayedimmediate cohort scored higher than the delayed cohort (3.84 vs. 2.94, $\mathrm{P}<0.01$ ).

Final aesthetic outcomes were assessed on a 2 -value scale; good and adequate. Patients were determined to have a "good" aesthetic outcome if the average, overall aesthetic score was between a 4 and 5. Patients that scored between 2 and 3.9 had an "adequate" overall aesthetic result. No patients scored less than 2 .
Table 4. Associations between overall aesthetic outcomes and patient or operative factors

\begin{tabular}{|lccc|}
\hline Variable & $\begin{array}{c}\text { Good result } \\
\text { (scored 4-5) }\end{array}$ & $\begin{array}{c}\text { Adequate result } \\
\text { (scored 2-3.9) }\end{array}$ & P-value \\
\hline Left breast reconstruction & $10 / 16$ & $6 / 16$ & 0.67 \\
Right breast reconstruction & $12 / 24$ & $12 / 24$ & 0.77 \\
DIEP & $10 / 24$ & $14 / 24$ & 0.2 \\
Muscle sparing TRAM (MS2) & $12 / 16$ & $4 / 16$ & 0.07 \\
Body mass index > 30 kg/m² & $10 / 22$ & $12 / 22$ & 0.99 \\
Smoking & $2 / 12$ & $10 / 12$ & 0.05 \\
30-Day postoperative complications & $0 / 4$ & $4 / 4$ & 0.19 \\
\hline $\begin{array}{l}\text { DIEP, deep inferior epigastric perforator flap; TRAM, transverse rectus abdominis } \\
\text { myocutaneous; MS, muscle sparing. }\end{array}$ & \multicolumn{4}{|l}{} \\
\hline
\end{tabular}

Given the small sample size, results were combined between the DR and DIR cohorts to uncover associations between patient or treatment variables and ultimate aesthetic outcomes regardless of the timing to a given reconstruction. Overall, 22 patients achieved a good overall aesthetic result and 18 patients achieved an adequate overall aesthetic result (Table 4). Smoking was the only variable found to have a statistically significant effect and was associated with lower overall aesthetic outcome scores. There were 12 patients (30\%) who reported a history of smoking within 3 months of their mastectomy. Two of these patients achieved a good aesthetic result following autologous breast reconstruction while 10 achieved an adequate result, $\mathrm{P}=0.05$. None of the patients reported an active smoking history at the time of their reconstruction. Alternatively, breast laterality, the type of autologous reconstruction (DIEP vs. muscle sparing [MS] TRAM), BMI over $30 \mathrm{~kg} / \mathrm{m}^{2}$, or the development of a complication within the initial 30-day postoperative period did not have a statistically significant impact on aesthetic outcomes. There were two postoperative complications that included a superficial cellulitis that was noted on post-surgical day nine and a seroma that developed 2 weeks following surgery. These were managed with oral antibiotic therapy and aspiration respectively. Within a given treatment cohort, associations between patient or treatment factors and aesthetic outcomes were explored (Table 5). For patients in the DR treatment group, there were no statistically significant differences in patient or treatment factors between those who achieved a good versus those who achieved an adequate aesthetic outcome. Alternatively, the time between radiation therapy completion and autologous breast reconstruction was found to significantly affect aesthetic outcomes in the DIR treatment algorithm. All patients who underwent an autologous reconstruction within 90 days of finishing PMRT scored lower in their overall aesthetic score compared to those who completed their reconstruction greater than 90 days from radiation therapy, $\mathrm{P}=0.04$. 
Table 5. Associations between overall aesthetic outcomes and patient or operative factors by treatment algorithm

\begin{tabular}{|c|c|c|c|}
\hline Variable & $\begin{array}{l}\text { Good result } \\
\text { (scored 4-5) }\end{array}$ & $\begin{array}{l}\text { Adequate result } \\
\text { (scored 2-3.9) }\end{array}$ & P-value \\
\hline \multicolumn{4}{|l|}{ Delayed reconstruction (DR) } \\
\hline Left breast reconstruction & $2 / 8$ & $6 / 8$ & 0.63 \\
\hline Right breast reconstruction & $4 / 12$ & $8 / 12$ & 0.7 \\
\hline DIEP & $4 / 10$ & $6 / 10$ & 0.99 \\
\hline MS2 & $2 / 10$ & $8 / 10$ & 0.37 \\
\hline Smoking & $0 / 6$ & $6 / 6$ & 0.52 \\
\hline Body mass index $>30 \mathrm{~kg} / \mathrm{m}^{2}$ & $6 / 16$ & $10 / 16$ & 0.99 \\
\hline 30-Day postoperative complications & $0 / 2$ & $2 / 2$ & 0.99 \\
\hline $\begin{array}{l}\text { Reconstruction within } 90 \text { days } \\
\text { from radiation }\end{array}$ & 0 & 0 & NA \\
\hline \multicolumn{4}{|l|}{ Delayed immediate reconstruction } \\
\hline Left breast reconstruction & $8 / 8$ & $0 / 8$ & 0.12 \\
\hline Right breast reconstruction & $8 / 12$ & $4 / 12$ & 0.68 \\
\hline DIEP & $12 / 14$ & $2 / 14$ & 0.13 \\
\hline MS2 & $4 / 6$ & $2 / 6$ & 0.99 \\
\hline Smoking & $2 / 6$ & $4 / 6$ & 0.20 \\
\hline Body mass index $>30 \mathrm{~kg} / \mathrm{m}^{2}$ & $2 / 6$ & $4 / 6$ & 0.20 \\
\hline 30-Day postoperative complications & $2 / 2$ & $0 / 2$ & 0.99 \\
\hline $\begin{array}{l}\text { Reconstruction within } 90 \text { days } \\
\text { from radiation }\end{array}$ & $0 / 6$ & $6 / 6$ & 0.04 \\
\hline \multicolumn{4}{|c|}{$\begin{array}{l}\text { NA, not applicable; DIEP, deep inferior epigastric perforator flap; TRAM, transverse } \\
\text { rectus abdominis myocutaneous; MS, muscle sparing. }\end{array}$} \\
\hline
\end{tabular}

\section{DISCUSSION}

Post-mastectomy radiation therapy has been associated with an increased incidence of complications in the setting of breast reconstruction [14-16]. Momoh et al. [16] demonstrated a $40 \%$ complication rate following delayed autologous breast reconstruction in the setting of PMRT while only $20 \%$ of patients developed a complication in the absence of radiation therapy, $\mathrm{P}=0.002$. One of the main adverse events in the radiated patients was incisional dehiscence that occurred in $11 \%$ of patients following radiation and in $3 \%$ of patients that were not radiated, $\mathrm{P}=0.05$. Despite the negative effects of PMRT on autologous and device-based reconstructions, autologous tissue has been reported to tolerate radiation more favorably than device-based reconstructions [17].

Post mastectomy radiation can also adversely affect aesthetic outcome following reconstruction. In a retrospective review, Roostaeian et al. [18] demonstrated significantly lower aesthetic scores as judged by a panel of blinded observers, in the setting of radiotherapy $(\mathrm{P}=0.047)$, breast size equal to or great than a $\mathrm{D}$ cup $(\mathrm{P}=0.018)$, and grade 2 ptosis $(\mathrm{P}=0.017)$ following immediate, implant-based reconstructions. The effect of radiation on aesthetic outcomes following delayed versus delayed-immediate autologous reconstructions remains uncertain. Proponents of the DIR opine that improved aesthetic results are obtained be- cause the natural breast landmarks such as the inframammary and lateral mammary fold are preserved, the breast pocket is maintained, and the additional native breast skin will enhance the ability to obtain a more natural breast shape and contour.

Based on our review of the literature and the PubMed database, this is the first study comparing aesthetic outcomes following delayed and delayed-immediate autologous reconstructions. The main findings included improvement in scar and skin quality in the DIR cohort for which there are several explanations. The importance of scar positioning and skin preservation are vital components for optimizing aesthetic outcome. The ability to preserve some of the native breast skin in the upper pole of the reconstructed breast will allow for a more inferiorly placed incision enabling the scars to be more optimally positioned and preserving natural breast aesthetics. In addition, the superior aspect of the flap can be positioned superiorly on the chest and under the remaining mastectomy skin leading to a more favorable chest wall to breast transition. Inferior pole contour is also improved following DIR reconstruction because less of the lower pole skin is excised thus preserving the natural inframammary fold and facilitating the creation of a more ptotic breast.

There are other factors that can affect aesthetic outcomes in the setting of mastectomy, radiation, and reconstruction. Variations in PMRT include the timing of radiation therapy, delivery method, boost dose administration, fractionation, and overall dose delivered. In particular, the timing between radiation and reconstructive surgery may affect ultimate outcomes. Recent investigations suggest that the optimal timing of a delayed autologous reconstruction is approximately 12 months following radiation [19]. Our study demonstrated aesthetic results were compromised when the reconstruction was completed within 3 months from the completion of radiation therapy. This may be the result of prolonged inflammatory changes to the skin following radiation that may adversely impact healing and scaring when the reconstruction is performed too early. Despite our observations, Momoh et al. [16] failed to identify a statistically significant difference in complications between patients who were reconstructed in less than six months, six to twelve months, or greater than twelve months from PMRT.

Smoking and obesity are known to increase complication rates in the setting of breast reconstruction; however, these co-morbidities are not absolute contraindications for breast reconstruction. In this study, smoking was demonstrated to significantly decrease aesthetic outcomes for both the DR and DIR cohorts. However, this study failed to identify a difference in outcomes relative to obesity. This is in contradiction to a previously published prospective analysis of 624 free-flap breast reconstructions that demonstrated higher rates of donor site wound healing 
complications in active smokers and greater numbers of total flap loss in obese patients [20]. Berry et al. [17] retrospectively reviewed autologous tissue and implant-based reconstructions in 1,037 patients and found no effect of smoking, hypertension, or chemotherapy on ultimate reconstructive outcomes. They were able to demonstrate, however, that age and a BMI over 30 were significantly associated with increased complications following prosthetic device-based reconstructions but not autologous reconstructions. In a retrospective review of outcomes following breast reconstruction and PMRT, Hirsch compared patients who underwent a delayed reconstruction and an immediate reconstruction but were unable to demonstrate any differences in complication rates based on age, BMI, or smoking status [21].

In conclusion, improved aesthetic outcomes can be obtained using a delayed-immediate reconstructive algorithm as compared to a delayed algorithm. Preservation of native mastectomy skin allows for improved skin/scar quality, breast contour, and overall aesthetic outcomes. This study is limited by the retrospective design as well as relatively small patients considered; however, associations between outcomes and the timing of the reconstruction relative to PMRT or smoking were identified. Further prospective evaluation would be useful to better understand the significance of increasing the time from radiation completion to ultimate autologous breast reconstruction on aesthetic outcomes.

\section{REFERENCES}

1. Fisher B, Anderson S, Bryant J, et al. Twenty-year follow-up of a randomized trial comparing total mastectomy, lumpectomy, and lumpectomy plus irradiation for the treatment of invasive breast cancer. N Engl J Med 2002;347:1233-41.

2. Fisher B, Dignam J, Wolmark N, et al. Lumpectomy and radiation therapy for the treatment of intraductal breast cancer: findings from National Surgical Adjuvant Breast and Bowel Project B-17. J Clin Oncol 1998;16:441-52.

3. Kronowitz SJ. Current status of autologous tissue-based breast reconstruction in patients receiving postmastectomy radiation therapy. Plast Reconstr Surg 2012;130:282-92.

4. Albino FP, Koltz PF, Ling MN, et al. Irradiated autologous breast reconstructions: effects of patient factors and treatment variables. Plast Reconstr Surg 2010;126:12-6.

5. Jhaveri JD, Rush SC, Kostroff K, et al. Clinical outcomes of postmastectomy radiation therapy after immediate breast reconstruction. Int J Radiat Oncol Biol Phys 2008;72:85965.

6. Wong JS, Ho AY, Kaelin CM, et al. Incidence of major corrective surgery after post-mastectomy breast reconstruction and radiation therapy. Breast J 2008;14:49-54.

7. Kronowitz SJ. Current status of implant-based breast reconstruction in patients receiving postmastectomy radiation therapy. Plast Reconstr Surg 2012;130:513e-23e.

8. Chang DW, Barnea Y, Robb GL. Effects of an autologous flap combined with an implant for breast reconstruction: an evaluation of 1000 consecutive reconstructions of previously irradiated breasts. Plast Reconstr Surg 2008;122:35662.

9. Crisera CA, Chang EI, Da Lio AL, et al. Immediate free flap reconstruction for advanced-stage breast cancer: is it safe? Plast Reconstr Surg 2011;128:32-41.

10. Kronowitz SJ, Robb GL. Radiation therapy and breast reconstruction: a critical review of the literature. Plast Reconstr Surg 2009; 124:395-408.

11. Tran NV, Evans GR, Kroll SS, et al. Postoperative adjuvant irradiation: effects on tranverse rectus abdominis muscle flap breast reconstruction. Plast Reconstr Surg 2000;106:313-7.

12. Kronowitz SJ, Hunt KK, Kuerer HM, et al. Delayed-immediate breast reconstruction. Plast Reconstr Surg 2004;113: 1617-28.

13. Kronowitz SJ, Lam C, Terefe W, et al. A multidisciplinary protocol for planned skin-preserving delayed breast reconstruction for patients with locally advanced breast cancer requiring postmastectomy radiation therapy: 3-year followup. Plast Reconstr Surg 2011;127:2154-66.

14. Hvilsom GB, Holmich LR, Steding-Jessen M, et al. Delayed breast implant reconstruction: is radiation therapy associated with capsular contracture or reoperations? Ann Plast Surg 2012;68:246-52.

15. Whitfield GA, Horan G, Irwin MS, et al. Incidence of severe capsular contracture following implant-based immediate breast reconstruction with or without postoperative chest wall radiotherapy using 40 Gray in 15 fractions. Radiother Oncol 2009;90:141-7.

16. Momoh AO, Colakoglu S, de Blacam C, et al. Delayed autologous breast reconstruction after postmastectomy radiation therapy: is there an optimal time? Ann Plast Surg 2012; 69:14-8.

17. Berry T, Brooks S, Sydow N, et al. Complication rates of radiation on tissue expander and autologous tissue breast reconstruction. Ann Surg Oncol 2010;17 Suppl 3:202-10.

18. Roostaeian J, Pavone L, Da Lio A, et al. Immediate placement of implants in breast reconstruction: patient selection and outcomes. Plast Reconstr Surg 2011;127:1407-16.

19. Baumann DP, Crosby MA, Selber JC, et al. Optimal timing of delayed free lower abdominal flap breast reconstruction after postmastectomy radiation therapy. Plast Reconstr Surg 
2011;127:1100-6.

20. Seidenstuecker K, Munder B, Mahajan AL, et al. Morbidity of microsurgical breast reconstruction in patients with comorbid conditions. Plast Reconstr Surg 2011;127:1086-92.
21. Hirsch EM, Seth AK, Dumanian GA, et al. Outcomes of tissue expander/implant breast reconstruction in the setting of prereconstruction radiation. Plast Reconstr Surg 2012;129:354-61. 